\title{
Comparación de medios de cultivos y procedimientos para detectar colonización por Streptococcus agalactiae en mujeres embarazadas
}

\author{
Tulia M. Díaz y Beatriz M. Nieves
}

Universidad de los Andes.
Mérida - Venezuela.
Facultad de Farmacia y Bioanálisis
Departamento de Bioanálisis Clínico
(TMD)
Departamento de Microbiología y Parasitología (BMN)

Recibido: 28 enero 2007 Aceptado: 14 enero 2008

Correspondencia a: Tulia Mariela Díaz tulia@ula.ve.

\section{Comparison between culture media and procedures to detect Streptococcus agalactiae in pregnant women}

Detection of Streptococcus agalactiae in pregnant women's vagina and rectum and intrapartum antibiotic prophylaxis administered to colonized women are currently recommended to prevent neonatal precocious infections by this organism. In turn, it is very important to select the culture media and adequate sample collection site for $S$. agalactiae detection in colonized women. To standardize this methods in laboratory, different culture media and procedures for $S$. agalactiae recovery in pregnant women with obstetric and gynecologic complications were compared. Vaginorectal and endocervical swab specimens were collected from 60 pregnant women. The first sample was placed onto selective Columbia blood agar directly and onto selective Todd-Hewitt broth incubated at $37^{\circ} \mathrm{C}$ and subcultured onto selective Columbia blood agar at 4 and 18 hours. The second sample was cultured on selective Columbia blood agar. Both culture media were incubated in a microaerophilic atmosphere at $37{ }^{\circ} \mathrm{C}$ from 24 to 48 hours. S. agalactiae was identified using conventional tests. 21 patients colonized with $S$. agalactiae were detected using vaginoanorectal samples. $19(31.7 \%)$ patients tested positive for S. agalactiae through the culture of specimens directly onto selective Columbia blood agar; 21 (35\%) and 20 (33\%) patients were found to be positive for S. agalactiae by the selective Todd-Hewitt broth at 4 and 18 hours, respectively. Only one patient tested positive for $S$. agalactiae in the endocervical tract. The results show that the three procedures followed for S. agalactiae recovery are effective. Nevertheless, the procedure in which the sample was placed directly onto selective Columbia blood agar permits reducing costs and the time for bacteria identification. On the other hand, the vaginoanorectal swab was the best sample to detect colonization by S. agalactiae in pregnant women.

Key words: Streptococcus agalactiae, vaginoanorectal colonization, selective Todd-Hewitt broth, selective Columbia blood agar.

Palabras clave: Streptococcus agalactiae, colonización vagino-ano-rectal, caldo selectivo Todd-Hewitt, agar sangre Columbia selectivo.

\section{Introducción}

$\mathrm{L}$ a detección de Streptococcus agalactiae en la vagina y/o el recto de las mujeres embarazadas y la administración de profilaxis antimicrobiana intraparto en las colonizadas, es el método más eficaz y recomendado para prevenir la infección neonatal precoz por este microorganismo ${ }^{10}$.

Numerosos estudios han documentado que los cultivos solamente de vagina o sólo del cérvix, son poco sensibles para detectar colonización por S. agalactiae. Existen evidencias de que los cultivos provenientes de hisopados tomados primero del tercio inferior de la vagina y luego del anorrecto tienen alta sensibilidad para la detección de S. agalactiae, incrementándose su detección en $25 \%$ sobre el cultivo vaginal solo ${ }^{26,36}$.

De igual manera, la elección del medio de cultivo para detectar $S$. agalactiae puede influir de forma importante en la eficacia de las medidas de prevención, ya que son diversos los medios de cultivo que se usan para este fin ${ }^{5}$. Los estudios refieren que la sensibilidad del cultivo se ve aumentada cuando se usa el caldo enriquecido selectivo como método de detección primaria $^{18}$. Al respecto, se han evaluado varias fórmulas de caldos enriquecidos selectivos para recuperar $S$. agalactiae, el más estudiado y usado ha sido el caldo Todd Hewitt (THB) con colistin $(10 \mu \mathrm{g} / \mathrm{ml})$ y ácido nalidíxico (15 $\mu \mathrm{g} / \mathrm{ml})$, conocido como caldo $\operatorname{Lim}^{13,14,22,23}$. 
Diversos investigadores señalan que el uso de este tipo de caldos aumenta hasta en 50\% la detección de $S$. agalactiae en mujeres colonizadas cuando es comparado con el cultivo sólo en placa directa ${ }^{2,13,23}$. Sin embargo, recientemente, Rauen y $\mathrm{col}^{27}$, describieron que el caldo enriquecido no selectivo (Todd Hewitt) posee una sensibilidad comparable a la del caldo enriquecido selectivo en la detección de colonización por $S$. agalactiae en mujeres embarazadas.

El Centro de Control y Prevención de Enfermedades en Atlanta (CDC), actualizó sus lineamientos para la prevención de enfermedad perinatal por S. agalactiae; el principal cambio fue la recomendación de realizar cultivo vaginal y rectal a todas las mujeres embarazadas entre las 35 y 37 semanas de gestación, administrando profilaxis intraparto a todas las portadoras. Estas recomendaciones obedecen a datos que demuestran la superioridad de las estrategias de prevención de infección neonatal basadas en cultivos, sobre las establecidas en función de la existencia de factores de riesgo ${ }^{10}$.

Con el propósito de estandarizar una metodología en el laboratorio que garantice la recuperación de $S$. agalactiae, se compararon diferentes medios de cultivos y procedimientos para la recuperación de este microorganismo en mujeres embarazadas con complicaciones gineco-obstétricas, que asistieron al Servicio de Emergencia de Obstetricia del Instituto Autónomo, Hospital Universitario de los Andes (I.A.H.U.L.A) en Mérida, Venezuela.

\section{Material y Métodos}

Estudio microbiológico. Se utilizó la metodología propuesta por el $\mathrm{CDC}^{8}$ modificada, la propuesta por García y $\operatorname{col}^{15}$, y la siembra directa de la muestra endocervial en medio selectivo. La metodología propuesta por el CDC recomienda colocar los hisopos con la muestra vagino-ano-rectal en el medio de transporte Stuart o Amies y luego inocularla en el caldo ToddHewitt selectivo, SBM o Lim. Incubar durante 18 a 24 horas y sub-cultivar a placas de agar sangre. En el presente estudio se realizó la siembra directa de la muestra vagino-ano-rectal en caldo Todd - Hewitt selectivo, con subcultivo a la 4 y 18 horas de incubación en agar sangre selectivo. También se realizó el cultivo directo de las muestras vagino-ano-rectales ${ }^{15}$ en agar sangre selectivo y el cultivo de las muestras endocervicales en agar sangre selectivo, como se describe en la Figura 1.

Muestra. Se procesaron 60 muestras de secreción vagino-ano-rectal y endocervical provenientes de mu- jeres embarazadas con complicaciones gineco-obstétricas y edad gestacional entre 26 y 32 semanas, quienes manifestaron por escrito su aceptación para ser incluidas en el estudio.

Cultivo. Las muestras de secreción vagino-anorectal se obtuvieron sin uso de espéculo, mediante tres hisopos de algodón estériles introducidos secuencialmente, primero en el tercio inferior de la vagina y luego se introdujo y rotó en ano-recto. Adicionalmente, con otro hisopo, se recolectó muestra endocervical.

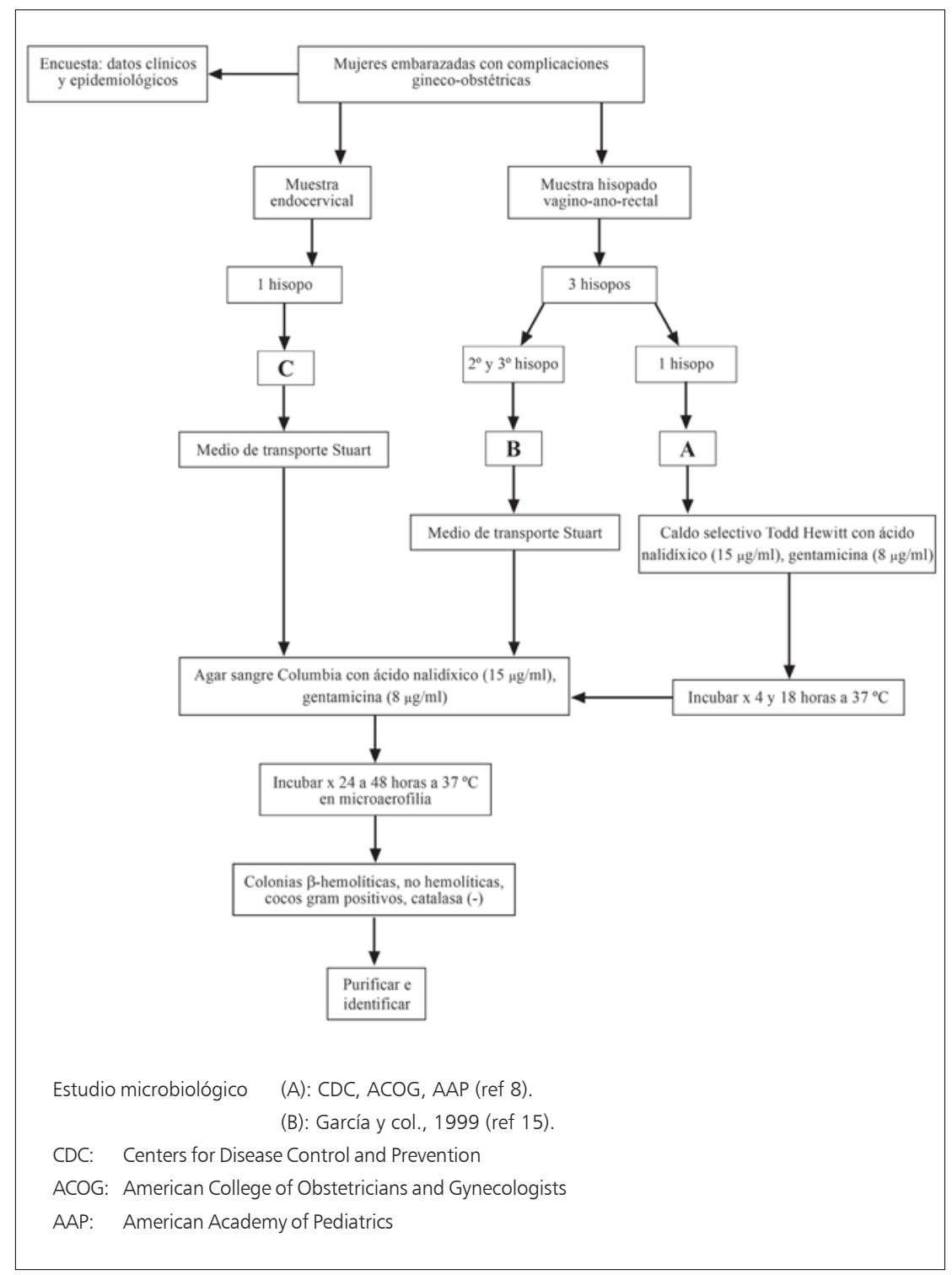

Figura 1. Estudio microbiológico propuesto por el CDC, ACOG, AAP, en 1996 con modificaciones (García y col., 1999). 
Figura 2. Porcentaje de recuperación de $\boldsymbol{S}$. agalactiae según el medio de cultivo utilizado para su detección en mujeres embarazadas con complicaciones gineco-obstétricas.

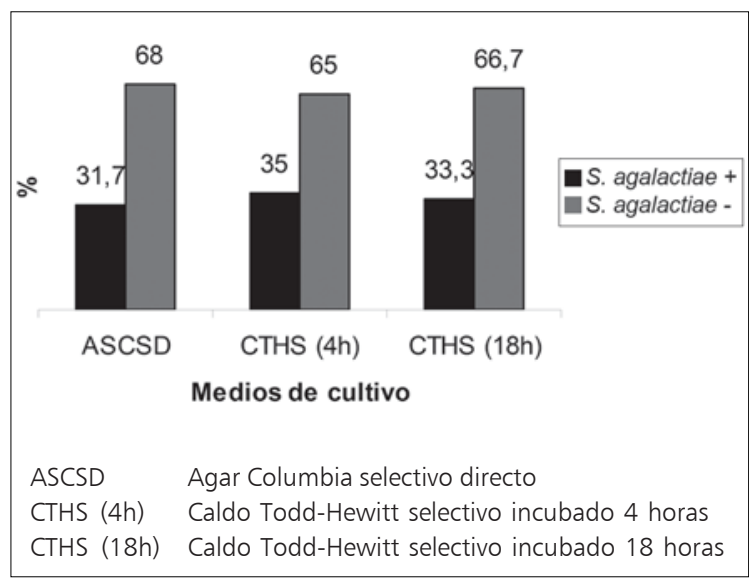

El primer hisopo se colocó en $5 \mathrm{ml}$ de caldo Todd Hewitt selectivo (CTHS) $[15 \mu \mathrm{g} / \mathrm{ml}$ de ácido nalidíxico y $8 \mu \mathrm{g} / \mathrm{ml}$ de gentamicina], el cual se incubó a $37^{\circ} \mathrm{C}$ y se sub-cultivó a las 4 y 18 horas en agar sangre Columbia selectivo [5\% de sangre humana, $15 \mu \mathrm{g} / \mathrm{ml}$ de ácido nalidíxico y $8 \mu \mathrm{g} / \mathrm{ml}$ de gentamicina] (ASCS) (CDC modificado).

Los hisopos restantes (segundo y tercero) con la muestra vagino-ano-rectal, se introdujeron en el medio de transporte Stuart, para realizar en el laboratorio la siembra directa en agar sangre Columbia selectivo $(\mathrm{ASCSD})^{15}$. La muestra endocervical se sembró en ASCS. Las placas de ASCSD y ASCS se incubaron a $37^{\circ} \mathrm{C}$ en atmósfera microaerofílica durante 24 a 48 horas. Una vez identificadas las colonias sugerentes de S. agalactiae, se determinó el grado de colonización en alta si se observaba crecimiento en el tercer y cuarto cuadrante de la placa de agar, y en baja si solamente había crecimiento en el primer y segundo cuadrante, de acuerdo a los criterios descritos por Krohn y $\operatorname{col}^{19}$. Se consideró colonización positiva cuando el microorganismo se recuperó de cualquiera de los procedimientos utilizados. La identificación de $S$. agalactiae se basó en pruebas convencionales previamente publicadas: tipo de hemólisis, tinción de Gram, prueba de catalasa, prueba de CAMP, hidrólisis del hipurato y crecimiento en bilis esculina ${ }^{20}$. Todas las cepas identificadas presuntivamente como $S$. agalactiae por las pruebas antes mencionadas, se confirmaron mediante la detección del antígeno específico de grupo, siguiendo las instrucciones del fabricante (Slidex Strep B- bioMerieux).

Análisis estadístico. Para evaluar la relación entre los tres medios se utilizó la prueba de $\chi^{2}$ con la incorporación de la corrección de Yates.

\section{Resultados}

De las 60 pacientes evaluadas, 21 (36,7\%) presentaron cultivo positivo para $S$. agalactiae en la muestra proveniente de secreción vagino-ano-rectal. La detección de $S$. agalactiae fue de la siguiente manera: $31,7 \%$ (19/60) se aislaron en el ASCSD, 35\% (21/60) en el CSTH 4 horas y 33,3\% (20/60) en el CSTH 18 horas. De los 21 cultivos positivos para S. agalactiae detectado en el CSTH $4 \mathrm{~h}$, 19 fueron positivos también en la placa directa de ASCSD. De los 20 cultivos positivos para $S$. agalactiae detectado en CSTH 18 horas, 17 fueron positivos en la placa de ASCSD. De las 21 pacientes colonizadas, sólo a una paciente se le detectó S. agalactiae en la muestra de secreción vagino-ano-rectal y endocervical simultáneamente. No se observó diferencia significativa en la recuperación de $S$. agalactiae en cultivos y procedimientos ensayados (Figura 2).

\section{Discusión}

Es importante detectar la colonización por $S$. agalactiae en mujeres embarazadas debido a las complicaciones gineco-obstétricas que puede ocasionar este microorganismo. Es frecuente la colonización de la vagina por $S$. agalactiae a partir del recto, y de esta zona, la bacteria migra al cérvix por vía ascendente, donde propicia la infección, la cual puede alterar el moco cervical llevando a rotura prematura de membranas e inicio prematuro del parto ${ }^{28}$.

Por otra parte, la colonización de los recién nacidos se produce durante el parto a partir del tracto genital materno colonizado o en el útero por vía ascendente, siendo la tasa de transmisión vertical de 50\%. Streptococcus agalactiae es el principal agente etiológico de sepsis neonatal precoz, la cual tiene una frecuencia entre 1 a $4 \backslash 1.000$ nacidos vivos y una letalidad estimada entre 5 y $20 \% \%^{3,9}$.

Los estudios de prevalencia de $S$. agalactiae en mujeres embarazadas muestran tasas de colonización que varían entre 5 y $25 \% \%^{34,35}$, dependiendo de la población estudiada, la ubicación geográfica, la región anatómica de obtención de la muestra (vaginal y/o anal) y el medio de cultivo utilizado (selectivo y no selectivo).

Algunos autores ${ }^{11,21,25}$ señalan que la colonización por $S$. agalactiae es poco frecuente en mujeres provenientes de países en vías de desarrollo; no obstante, en el presente estudio, la colonización vagino-anorectal por S. agalactiae fue de $36,7 \%$, resultado que se correlaciona con el porcentaje reportado en otros estudios realizados en mujeres embarazadas ${ }^{15,17,27,29,30,33}$.

En nuestro medio, no está clara la incidencia de infección neonatal por S. agalactiae, ni el peso que 
tiene sobre la morbi-mortalidad neonatal; en este trabajo se evidenció porcentajes altos de colonización por $S$. agalactiae en muestras vagino-ano-rectales provenientes de mujeres embarazadas, cifras que se asemejan más a la de países industrializados, por lo que pudiéramos pensar que la tasa de transmisión y enfermedad en los bebés también debe ser importante en nuestro país; desafortunadamente, no se cuenta con estadística ni estudios que respalden tal afirmación debido a que no existen programas de vigilancia de esta infección en neonatos. Esta diferencia en las cifras de colonización puede explicarse, en parte, por la metodología utilizada, el tipo de muestra (hisopado vagino-ano-rectal) y la incorporación de cultivos selectivos, que aumentaron la recuperación de dicho microorganismo.

Con respecto al tipo de muestra, en la mayoría de las mujeres colonizadas se detectó el microorganismo únicamente de hisopado vagino-ano-rectal, sólo en uno de estos casos se recuperó $S$. agalactiae a partir de la muestra endocervical, encontrándose además en abundante cantidad. Este resultado difiere de lo reportado por otros investigadores, quienes encontraron colonización por $S$. agalactiae en muestras vaginales y/o endocervicales ${ }^{4,28}$, hallazgo que podría deberse a que la muestra no fue enriquecida en caldo selectivo y a que la carga bacteriana en esta localización es menor. Nuestros hallazgos ratifican la importancia de la obtención de muestra de la región vagino-ano-rectal, como método de pesquisa para evitar el riesgo de transmisión vertical al neonato al no recibir profilaxis inmediata adecuada.

Por otra parte, la técnica de cultivo aceptada universalmente para la detección de colonización por $S$. agalactiae en mujeres embarazadas ha sido la propuesta por el $\mathrm{CDC}^{8}$, quien recomienda el uso de medios líquidos enriquecidos selectivos (caldo Lim o Todd Hewitt con ácido nalidíxico y gentamicina) para maximizar la recuperación de este microorganismo. En este sentido, en el presente estudio, utilizar CTHS sub-cultivado a las 4 horas de incubación, además de las 18 horas, como lo recomienda el CDC, permitió acelerar el diagnóstico.

Existen diferencias en la utilización de medios sólidos y caldos selectivos para incrementar la recuperación de $S$. agalactiae de hisopados vagino-anorectales de mujeres embarazadas ${ }^{1,5,6,10,12,15,16,24,31}$. En este trabajo, tanto el uso del CSTH sub-cultivado a las $4 \mathrm{y}$ 18 horas de incubación, como el ASCS con siembra directa, mostraron igual efectividad para la recuperación de S. agalactiae a partir de las muestras analizadas. Sin embargo, se pudo observar el sobrecrecimiento de otros microorganismos en las placas de ASCS subcultivado a partir de los caldos selectivos, indepen- dientemente de las horas de incubación, especialmente Enterococcus sp. y Staphylococcus sp, lo cual dificultó el aislamiento de $S$. agalactiae. De hecho, en dos oportunidades $S$. agalactiae fue recuperado de la placa de ASCSD y no se pudo recuperar a partir del subcultivo del caldo Todd Hewitt incubado durante 18 horas. Estos resultados difieren de los hallazgos de Bosh y $\mathrm{col}^{4}$, Rauen y $\mathrm{col}^{27}$, quienes refieren baja sensibilidad para detectar S. agalactiae utilizando siembra directa en ASCS.

Se estima que la aplicación de estrategias de prevención basadas en cultivo permite prevenir más de $90 \%$ de los casos de infección neonatal por S. agalactiae, mientras que las basadas únicamente en factores de riesgo previenen 50 a $60 \%$ de los casos. En este sentido, es importante que los laboratorios realicen una revisión cuidadosa de los protocolos de detección de $S$. agalactiae, de manera de implementar los más sensibles y que se puedan desarrollar en todos los laboratorios microbiológicos, sin inconvenientes y con mínimos costos ${ }^{13,27}$.

Los resultados obtenidos permiten concluir que los tres procedimientos ensayados presentaron igual efectividad para la recuperación de S. agalactiae; sin embargo, con el uso de ASCSD se disminuyen los costos y el tiempo de identificación de dicho microorganismo. Por otra parte, el hisopado vagino-ano-rectal resultó la muestra más adecuada para detectar colonización por S. agalactiae en mujeres embarazadas.

\section{Resumen}

Introducción: La detección de Streptococcus agalactiae en la vagina y/o el recto de las mujeres embarazadas y la administración de profilaxis antimicrobiana intraparto en las colonizadas, es el método recomendado para prevenir la infección neonatal precoz por este patógeno. En consecuencia, es importante seleccionar los medios de cultivos y el sitio de toma de muestra más adecuado para la detección de $S$. agalactiae en mujeres colonizadas. Objetivo: Comparar diferentes medios de cultivos y procedimientos para la recuperación de $S$. agalactiae en mujeres embarazadas con complicaciones gineco-obstétricas. Metodología: Se tomaron hisopados vagino-ano-rectales y endocervicales de 60 mujeres embarazadas. Con la primera muestra se realizó cultivo directo en agar sangre Columbia selectivo (ASCSD), y caldo selectivo Todd Hewitt (CSTH) incubados a $37^{\circ} \mathrm{C}$, y subcultivos a las 4 y 18 horas en agar sangre Columbia selectivo (ASCS). La segunda muestra se cultivó en ASCS. El ASCSD y ASCS se incubaron en atmósfera microaerófíla a $37^{\circ} \mathrm{C}$ durante 24 a 48 horas. La identificación de S. agalactiae 
se realizó mediante pruebas convencionales. Resultados: Utilizando hisopado vagino-ano-rectal se detectaron 21 pacientes colonizadas con S. agalactiae, de la siguiente manera: 19 (31,7\%) en el ASCSD, 21 (35\%) en el CSTH a las 4 horas y 20 (33,3\%) a las18 horas. De las 21 pacientes colonizadas sólo a una paciente se le detectó $S$. agalactiae en la muestra de secreción vagino-ano-rectal y endocervical simultáneamente.
Conclusión: Los tres procedimientos ensayados presentaron igual efectividad para la recuperación de $S$. agalactiae; sin embargo, con el uso del ASCSD, se disminuyen los costos y el tiempo de identificación de dicho microorganismo. Por otra parte, el hisopado vagino-ano-rectal resultó ser la muestra más idónea para detectar colonización por S. agalactiae en mujeres embarazadas.

\section{Referencias}

1.- Acikgoz Z, Turhan N, Gamberzade S, Gocer $S$. Increasing the detection rate of group B streptococcal carriers by nonselective cervico-vaginal culture accompanied with selective vaginoanorectal one. Diagnostic Microbiol Infect Dis 2003; 46: 69-71.

2.- Altaie S, Dryja D. Detection of group B Streptococcus. Comparison of solid and liquid culture media with and without selective antibiotics. Diagnostic Microbiol Infect Dis 1994; 18: 141-4.

3.- Belmar C, Abarzúa F, Becker J, Guzmán A M, García P, Oyarzún E. Estudio de sensibilidad antimicrobiana de 183 cepas de Streptococcus agalactiae aisladas en región vagino perineal de embarazadas en el tercer trimestre. Rev Chil Obstet Ginecol 2002; 67: 106-09.

4.- Bobitt J, Damato J, Sakakini J. Perinatal complications in group B streptococcal carrier: A longitudinal study of prenatal patients. Am J Obstet Gynecol 1985; 151: 711.

5.- Bosch J, Martín R, Jiménez, M. Estudio comparativo de tres medios de cultivos para detectar colonización por estreptococo del grupo B en la mujer embarazada. Enferm Infecc Microbiol Clin 2003; 21: 346-9.

6.- Bosch J, Murillo S, Rico M, Salgado, M. Utilidad de un medio selectivo disco-caldo para la detección de estreptococo del grupo B en la vagina. Enferm Infec Microbiol Clin 1998; 6: 83-4.

7.- Brian C, Yancey M, Uyehara C, Nauschuetz W. Effect of delayed inoculation of selective media antenatal detection of group B Streptococci. Obstet Gynecol 1998; 92: 923-5.

8.- Centers for Disease Control and Prevention. Prevention of perinatal group $B$ streptococcal disease: A public health perspective. Morb Mortal Wkly Rep 1996; 44: RR-7.

9.- Centers for Disease Control and Prevention. Adoption of hospital policies for prevention of perinatal group B streptococcal disease.
United States, Morb Mortal Wkly Rep 1998; 47: 665-70.

10.- Centers for Disease Control and Prevention. Prevention of perinatal group B streptococcal disease. Morb Mortal Wkly Rep 2002; 51: 1-22.

11.- Collins T, Calderón M, Gilman R, Vivar A, Charache, P. Group B streptococcal colonization in a developing country: Its association with sexually transmitted disease and socioeconomic factors. Am J Trop Med Hyg 1998; 59: 633-6.

12.- Dunne W, Holland-Staley C A. Comparison of NNA agar culture and selective broth culture for detection of group B streptococcal colonization in women. J Clin Microbiol 1998; 36: 2298-300.

13.- Elsayed S, Gregson D, Church D. Comparison of direct selective versus nonselective agar media plus LIM broth enrichment for determination of group B Streptococcus colonization status in pregnant women. Arch Pathol Lab Med 2003; 127: 718-20.

14.- Fenton L, Harper M. Evaluation of colistin and nalidix acid in Todd Hewitt broth for selective isolation and group B Streptococci. J Clin Microbiol 1979; 9: 176-9.

15.- García E, Rodríguez M, Bartolomé R, Borjano B, Cabrero l, Andreu A. Evaluation of the Granada agar plate for detection of vaginal and rectal group B Streptococci in pregnant women. J Clin Microbiol 1999; 37: 2648-51.

16.- Gupta Ch, Briski L. Comparison of two culture media and three sampling techniques for sensitive and rapid screening of vaginal colonization by group B Streptococcus in pregnant women. J Clin Microbiol 2004; 42: 3975-7.

17.- Hickman M, Rench A, Ferrieri P, Baker C. Changing epidemiology of group $\mathrm{B}$ streptococcal colonization. Pediatrics 1999; 104: 203-9.

18.- Hillier S, Schuhat A. Preventing neonatal group B streptococcal disease: The role of the clinical microbiology laboratory. Clin Microbiol Newslett 1997; 19: 113-5.

19.- Krohn M, Hillier S, Baker, C. Maternal peripartum complications associated with vaginal group B Streptococci colonization. J Infect Dis 1999; 179: 1410-5.

20.- Koneman E, Allen S, Janda W, Schreckenberger P, Winn W. Diagnóstico Microbiológico. Texto y Atlas a color. 5 ed. Buenos Aires (Argentina), Médica Panamericana; 1999.

21.- Sad Larcher J, Capellino F, De Giusto R, Travella C, Gómez F, Kreiker G, et al. Colonización por Streptococcus del grupo B durante el embarazo y prevención de enfermedad neonatal. Medicina 2005; 65: 201-6.

22.- Nguyen T, Gauthier D, Myles T, Nuwayhid B, Viana M, Schreckenberger P. Detection of group B Streptococcus: Comparison of an optical immunoassay with direct plating broth-enhanced culture methods. J. Matern Fetal Med 1998; 7: 1726.

23.- Orsello C, Dommermuth R. Maximizing neonatal early onset group B streptococcal disease prevention with universal culture screening at 35-37 weeks: a comparison of GBS detection rates between LIM broth and CAN culture media. Fam Med 2003; 35: 411-3.

24.- Overman S, Eley D, Jacobs B, Ribes J. Evaluation of methods to increase the sensitivity and timeliness of detection of Streptococcus agalactiae in pregnant women. J Clin Microbiol 2002; 40: 4329-31.

25.- Platt M, Gilson G. Group B streptococcal disease in the perinatal period. Am Fam Physician 1994; 49: 434-42.

26.- Quinlan J, Hill A, Maxwell B, Boone S, Hoover F, Lense J. The neccessity of both anorectal and vaginal cultures for group B Streptococcus screening during pregnancy. J Fam Pract 2000; 49: 447-8.

27.- Rauen N, Wesenberg E, Cartwright Ch. Comparison of selective and nonselective broth media for the detection of vaginal and ano-rectal colonization with group B Streptococcus. Diagnostic Microbiol Infect Dis 2005; 51: 9-12.

28.- Regan J, Chao S, James L. Premature rupture membranes, preterm delivery, and group B 
streptococcal colonization of mother. Am J Obstet Gynecol 1981; 141: 184-6.

29.- Regan J, Klebanoff $M$, Nugent $R$, and vaginal infections and prematury study group. For the Vaginal Infectious and Prematurity Study Group. The epidemiology of group B streptococcal colonization in pregnancy. Obstet Gynecol 1991; 77: 604-10.

30.- Riera L, Benavides G, Morillo N. Colonización por Streptococccus grupo B en embarazadas a término y recién nacidos en una comunidad de Venezuela. Enferm Infec Microbiol Clin 1993; 11: 295-8.

31.- Sayahtaheri S, Dryja D. Detection of group B Streptococcus: Comparison of solid and liquid culture media with and without selective antibiotics. Diagn Microbiol Infect Dis 1994; 18: 141-4.

32.- Schuchat A, Wenger J. Epidemiology of group B streptococcal disease. Risk factors, prevention strategies, and vaccine development. Epidemiol Rev 1994; 16: 374-402.

33.- Suara R, Adegbola R, Baker C, Secka O, Mulholland E, Greenwood B. Carriage of group B Streptococci in pregnant Gambian mothers and their infants. J Infect Dis 1994; 70: 1316-9.

34.- Valdés E, Pastene C, Garau M, Catalán J, Candia P, Juárez G, et al. Prevalencia de colonización por Streptococccus agalactiae (grupo B) en el tercer trimestre de embarazo. Rev Chil Obstet Ginecol 2003; 68: 305-8. 35.- Valdés E, Pastene C, Morales A, Gutiérrez B, Canales A, Martínez P, et al. Prevalencia de colonización por Streptococccus agalactiae (grupo B) durante el embarazo pesquisado en medio de cultivo selectivo. Rev Chil Obstet Ginecol 2004; 69: 132-5

36.- Yancey M, Duff P, Kurtzer T, Trentzen B, Kubilis, P. Peripartum infection associated with vaginal group B streptococcal colonization. Obstet Gynecol 1994; 84: 816-9. 